\title{
CAMBIOS AMBIENTALES EN LOS SISTEMAS FLUVIALES: NUEVAS METODOLOGÍAS, DIVERSIDAD DE CASOS E IMPLICACIONES PARA LA GESTIÓN
}

\section{INTRODUCCIÓN}

Entre los sistemas naturales que activan, dirigen y regulan el funcionamiento del planeta, los sistemas fluviales destacan por su intensa dinámica. Los cursos de agua, formando densas y complejas redes jerárquicas de cauces que cubren todos los territorios continentales, trabajan en la evacuación y el transporte de agua, sedimento y nutrientes desde cada lugar concreto hasta el mar, trabajo que es más intenso y de máxima eficacia en procesos de crecida (Ollero, 2017). En esta tarea permanente, aunque sometida a múltiples factores y complejas fluctuaciones, todos los cursos fluviales aportan funciones hidrogeomorfológicas y servicios ecosistémicos, así como geodiversidad y biodiversidad, y en conjunto conforman una función clave en el equilibrio del planeta, por cuanto dan continuidad y completan los ciclos hidrológicos, geomorfológicos y biogeoquímicos (Clarke et al., 2003).

La dinámica fluvial es la manifestación en el espacio y en el tiempo de este funcionamiento complejo (Rhoads, 2020), y debe ser apreciada también como un enorme valor ambiental. Por eso los cursos de agua, siempre dinámicos, son excelentes indicadores de cambios ambientales (Williamson et al., 2008; Macklin \& Woodward, 2009). La geomorfología fluvial es una ciencia ambiental que identifica esos cambios, los cuantifica y los interpreta, tratando de explicar sus causas y sus tendencias. Y todo ello en una red fluvial compleja con una enorme variedad de tipos de cursos de agua y de condiciones ambientales cambiantes (García et al., 2021).

El gran reto, lo más difícil de la geomorfología fluvial es encontrar, explicar y valorar los factores y procesos de cada cambio detectado (Sear \& Newson, 2003); en suma, ser capaces de definir en qué

a Departamento de Geografía, Universitat de València. Avda. Blasco Ibáñez, 28. 46010 València. francisca. segura@uv.es. https://orcid.org/0000-0002-7969-4740.

b Departamento de Geografía y Ordenación del Territorio, Universidad de Zaragoza. C/ Pedro Cerbuna, 12. 50009 Zaragoza. aollero@unizar.es. https://orcid.org/0000-0002-9745-5866. 
medida los cambios ambientales responden a factores naturales y/o antropogénicos y, además, si esos factores son locales, globales o proceden de otros sectores de la cuenca (Wyżga et al., 2012). Esta tarea tan compleja requiere trabajar a diferentes escalas y con diversas metodologías y técnicas. Requiere además el análisis de casos concretos para poder comparar, verificar y extraer conclusiones generales o identificar contrastes regionales.

\section{LOS CAMBIOS AMBIENTALES RECIENTES Y SUS IMPLICACIONES PARA LA GESTIÓN}

Durante los dos últimos siglos, los ríos han sufrido una importante metamorfosis tanto en las cuencas como en sus cauces. El flujo y el suministro de sedimentos han fluctuado a lo largo del tiempo, provocando ajustes continuos en el cauce a través de la erosión y la deposición de sedimentos. El carácter universal de estos impactos ha permitido observarlos a escala planetaria, en diferentes ambientes climáticos y con condicionantes sociales diversos.

Desde la segunda mitad del siglo xix hasta la actualidad se han observado en los ríos cambios importantes de diferente signo (Scorpio \& Piégay, 2021). En una primera fase, que llega hasta la segunda mitad del siglo xx, la deforestación, el cultivo en laderas con pendientes pronunciadas y el pastoreo extensivo causaron una fuerte erosión. La coincidencia en el tiempo con el final de la Pequeña Edad del Hielo y un periodo de importantes crecidas (Benito et al., 2008; Llasat, 2021), provocaron un incremento del caudal y de la carga sedimentaria, que se tradujeron en unos ríos agradacionales, con una morfología braided y una elevada movilidad lateral.

Por el contrario, a mediados del siglo xx, en una segunda fase, se produjo un cambio de tendencia que ha durado hasta finales del siglo xx. La despoblación de las zonas montañosas y el abandono de las tierras agrícolas y de la ganadería extensiva provocaron una recolonización del bosque y del matorral (Liébault \& Piégay, 2001; Garófano-Gómez et al., 2013; García Ruiz et al., 2015; Segura-Beltran \& Sanchis-Ibor, 2013). A ello se sumaron actuaciones antrópicas como la construcción de presas, la reforestación, las obras de control, la canalización de ríos o la extracción de gravas (Liébault \& Piégay, 2002; Surian \& Rinaldi, 2003; Surian et al., 2009; Batalla, 2003; Martin-Vide et al., 2010; García Ruiz \& Lana-Renault, 2011; Ollero et al., 2015; Calle et al., 2017; Sanchis-Ibor et al.,2017; Segura-Beltran $\&$ Sanchis-Ibor, 2020; Ollero et al., coords., 2021), que tuvieron un profundo impacto en los ríos, provocando un importante déficit hidrosedimentario. El resultado fue una incisión y un estrechamiento de los cauces acompañados de la invasión de la vegetación en el propio cauce.

La interacción entre la vegetación y la morfodinámica fluvial ha sido ampliamente estudiada en ríos perennes con lechos de gravas (Gurnell et al., 2009, 2012, 2015; Martínez-Fernández et al., 2016; García de Jalón et al., 2020), donde se ha documentado su importancia en la estabilización de las orillas, la fijación de los canales o su papel en la formación y fijación de las barras. En ríos efímeros o semipermanentes, algunos trabajos han demostrado que la influencia de la vegetación en la dinámica fluvial depende en gran medida de las variaciones de la secuencia temporal de las crecidas (Hooke \& Mant, 2002; Sandercock et al., 2007; Sanchis-Ibor et al., 2019; Sanchis-Ibor \& Segura-Beltran, 2020). 
En la mayoría de los ríos, la segunda fase se ha alargado hasta la actualidad; sin embargo, en algunas regiones europeas, se ha identificado una tercera fase que se inicia con el siglo xxi. Entre los años 2000 y 2010, algunos ríos han experimentado una inversión en sus trayectorias, consistente en el ensanchamiento del cauce, que se ha vuelto multicanal, y una ligera agradación. La ocurrencia de grandes crecidas fue una de las causas de esta recuperación, aunque en la mayoría de los casos se asoció al cese de la extracción de áridos (Segura-Beltran \& Sanchis-Ibor, 2018; Scorpio \& Piégay, 2021). Esta tendencia hacia la estabilidad o a un ligero ensanchamiento de los ríos no es universal, sino que presenta claros contrastes regionales; incluso en aquellos casos en los que se ha producido, tiene un carácter temporal, que no ha compensado la tendencia al estrechamiento y la incisión a largo plazo.

Comprender todos los cambios ambientales ocurridos en los últimos siglos es fundamental para la gestión de los ríos. A partir de la promulgación de la Directiva Marco del Agua 2000/60/CE (Comisión Europea, 2000), se ha producido un cambio de paradigma en la gestión y se ha despertado un cierto interés por la restauración fluvial, que ha desembocado en el desarrollo de la Estrategia Nacional de Restauración de Ríos en España. Aunque ya no se discute la necesidad y los beneficios de mantener los ríos en un buen estado ecológico, todavía existe una cierta controversia sobre el significado y la aplicación de este concepto (Ollero, 2011, 2020). La idea de restaurar los ríos y devolverlos a su condición prístina se ha mostrado como algo irreal, dado que no existe tal condición (Dufour \& Piégay, 2009). Como se ha mostrado anteriormente, los ríos del siglo xix son una quimera imposible de reproducir con las condiciones ambientales actuales. Es más, aquellos ríos fueron consecuencia de una intensa alteración antrópica sobre las cuencas, aunque poco importante sobre los cauces. Por el contrario, los ríos de la segunda mitad del siglo xx son consecuencia de una renaturalización de las cuencas y una fuerte alteración antrópica de los cauces. Ninguna de esas condiciones se puede recuperar y por eso se plantea la necesidad de utilizar las trayectorias de los ríos y sus tendencias como paso previo a cualquier posible restauración y huir de intentar replicar ríos que no volverán porque las condiciones ambientales ya no son las mismas.

Pero, además, existen todavía muchas incógnitas en el conocimiento de los ríos. En particular, el funcionamiento de la dinámica hidrosedimentaria y sus interacciones con la fauna y la flora que los habitan. La complejidad de los sistemas fluviales y, en concreto, de las relaciones biofísicas que en estos se establecen, debería obligar a la administración a tratarlos de forma holística y respetando los procesos naturales. Quizás la mejor solución para mantener nuestros ríos en las mejores condiciones radique en eliminar al máximo las actuaciones directas sobre los cauces y utilizar su capacidad de resiliencia para conseguir la autorrestauración (Segura-Beltran, 2014; Ollero, 2015; Segura-Beltran et al., 2021; Ollero et al., 2021). Permitir que el río trabaje según sus posibilidades sin intervenir activamente puede ser una buena estrategia, aunque no se avenga con los principios de restauración activa -una nueva alteración de los sistemas fluviales- que propugnan los gestores (Ollero et al., 2020; García et al., 2021). Por ello, es necesario tener un mejor conocimiento del comportamiento de los ríos en el pasado para poder proyectar las tendencias de su propia trayectoria hacia el futuro. De ahí la necesidad de realizar estudios con escalas temporales y espaciales variadas y aplicar nuevas técnicas para tener un mejor conocimiento de nuestros ríos. 


\section{CONTENido DEL MONOGRÁFICO}

En este número se recopilan once artículos que, desde diferentes perspectivas, con variadas metodologías, a distintas escalas y en diversos tipos fluviales, estudian la dinámica fluvial buscando respuestas al gran reto de comprender los cambios ambientales. Los once artículos pueden agruparse en tres grandes líneas: i) trabajos principalmente metodológicos (Díaz Alba et al., 2021; Díez Herrero et al., 2021; García \& Pérez Alberti, 2021; Gómez Gutiérrez et al., 2021 y Rabanaque et al., 2021), ii) análisis de cambios ambientales fundamentados en respuestas a impactos (Prats-Puntí et al., 2021; Ibisate et al., 2021 y Marco et al., 2021) y iii) estudios que inciden especialmente en las implicaciones para la gestión (Batalla, 2021; Benito et al., 2021 y Vidal-Abarca et al., 2021). Se abordan desde diferentes enfoques los cambios ambientales en cauces, tanto en ríos como en cursos efímeros, destacando también el interés por los riesgos asociados a las avenidas. Las áreas de estudio son diversas: Galicia, País Vasco, Pirineos, Canarias, Gredos, Madrid y, en el ámbito más claramente mediterráneo, las Bardenas de Navarra, Barcelona, Castelló, Alacant y Murcia.

En el primer bloque, de propuestas metodológicas, el trabajo de Díaz Alba et al. (2021) plantea el uso de la tecnología LiDAR, la clasificación de imágenes y las herramientas SIG para el seguimiento de acciones de restauración. En concreto, se expone la metodología empleada para analizar la evolución del corredor ripario tras la eliminación de un azud en el río Lozoya. Se cuantificaron los cambios en variables dendrométricas (altura, diámetro y biomasa) y dasométricas (fracción de cabida cubierta FCC) a partir de dos coberturas LiDAR PNOA (años 2010 y 2016) y de la clasificación de imágenes RGB PNOA. Los resultados confirmaron el crecimiento general esperado de la masa durante el periodo transcurrido entre los dos vuelos, tal como había sido observado en las variables (altura, diámetro, biomasa y densidad). También se detectaron cambios en la distribución de las clases de altura y diámetro, aumentando los pies de tamaños medios y reduciéndose los de tamaños extremos. La FCC disminuyó considerablemente tras la demolición del azud en las zonas más activas próximas al cauce principal, con un incremento importante de nuevos espacios abiertos. La metodología ha permitido valorar los cambios producidos en la estructura del corredor sin disponer de un muestreo de campo previo a la demolición. El ajuste de los datos medidos y estimados avala la calidad de la nube de puntos LiDAR y permite explorar las respuestas del corredor ante actuaciones de mejora de la conectividad longitudinal, tratamientos sobre la masa arbolada, plantaciones y otras actuaciones ligadas a proyectos de restauración, por lo que esta metodología puede suponer una herramienta eficaz y automatizable.

Díez Herrero et al. (2021) muestran el gran potencial de la dendrogeomorfología y la liquenometría para la reconstrucción y el análisis de avenidas torrenciales pretéritas, discuten las ventajas y limitaciones de estas técnicas y presentan ejemplos de aplicación en diferentes sistemas fluviales. Los resultados obtenidos son dataciones de eventos y mediciones de paleoniveles, área inundada, tiempo de permanencia, calados, velocidades y caudales generadores, lo que permite mejorar el análisis de frecuencias y la cuantificación de las magnitudes de avenidas pretéritas. Todo ello resulta especialmente útil donde no existe otro tipo de datos o donde los registros son cortos o discontinuos, como en zonas de montaña sin estaciones meteorológicas o aforos. El artículo explica las dos técnicas con detalle y se centra en 
tres casos de estudio concretos: barranco de las Angustias en el P. N. de la Caldera de Taburiente (La Palma), el arroyo Cabrera en Venero Claro (Ávila) y el barranco de Portainé (Pirineo de Lleida), en los que los autores cuentan con experiencia de trabajo, habiendo supuesto iniciativas pioneras, incluso a nivel internacional, y ejemplos para su extensión a otros territorios y problemáticas.

García y Pérez Alberti (2021) abordan la identificación y caracterización de los ríos en roca en Galicia a partir de sistemas de información geográfica y mediante el análisis de tres variables topo-geomorfológicas (litología, pendiente y densidad de fracturas). Los resultados obtenidos arrojan una primera cartografía de probabilidad de presencia de ríos en roca para más de $30.000 \mathrm{~km}$ de cauces, de los que un $37 \%$ de la red tiene una probabilidad alta de ser río en roca y un $21 \%$ una probabilidad baja o nula. Para validar los resultados se empleó la red de drenaje del Geoparque Mundial de la UNESCO Montañas del Courel. A partir de la distribución espacial obtenida, el trabajo ofrece una interesante aportación de utilidad para los organismos de cuenca y propone el establecimiento de protocolos de actuación que consideren las características de los ríos en roca, por su singularidad y repercusión en la dinámica, en la gestión de las masas de agua (Directiva 2000/60/CE), así como la necesidad de potenciar figuras de protección basadas en la geodiversidad, como por ejemplo las reservas naturales fluviales. En suma, este artículo combina aspectos metodológicos con objetivos de gestión.

Gómez Gutiérrez et al. (2021) analizan el potencial de las imágenes aéreas históricas y la fotogrametría automatizada para elaborar modelos 3D y cuantificar cambios morfológicos, centrando el estudio en cauces efímeros mediterráneos. Se seleccionaron tramos de las ramblas de Algeciras y Valdelentisco (cuenca del Segura) y del barranco de Tudela (cuenca del Ebro). Se emplearon fotogramas del vuelo americano B (1956) y del vuelo interministerial (1973-1986), junto con puntos de apoyo naturales registrados con un sistema de posicionamiento global (GNSS) para alimentar técnicas de fotogrametría automatizada (Structure-from-Motion $\mathcal{E}$ Multi-View Stereo) y producir nubes de puntos, modelos digitales de superficie y ortofotografías. Fue necesario adaptar el flujo de trabajo fotogramétrico convencional a las características de los fotogramas. Los productos cartográficos elaborados mediante fotogrametría automatizada alimentada con fotogramas aéreos históricos del vuelo americano B y el interministerial mostraron precisiones métricas. El vuelo interministerial dio lugar a productos cartográficos de mayor precisión y calidad, pero su geometría de la toma derivada de una menor altura de vuelo resultó en nubes de puntos con una cobertura menos homogénea que las obtenidas a partir del vuelo americano B. El trabajo concluye que el procesado multitemporal es una alternativa a la toma de GCP para la estimación de cambios a partir de fotografías aéreas históricas y fotogrametría automatizada.

Rabanaque et al. (2021) desarrollan un análisis automatizado de ortofotografías y técnicas de machine learning aplicado al estudio gemorfológico de cauces efímeros, con el objetivo de impulsar nuevos avances y aplicaciones de la geomorfología fluvial en el análisis medioambiental y el uso de los indicadores morfo-sedimentarios en la evaluación de los ríos. Estos indicadores son esenciales para el desarrollo de la Directiva 2000/60/CE en los ríos efímeros mediterráneos. La combinación de teledetección con técnicas de machine learning permite caracterizar las diferentes superficies fluviales de manera automática y objetiva, lo que supone un avance respecto a la fotointerpretación manual, que presenta una mayor carga de interpretación subjetiva. Las ortofotografías de alta resolución (0,25 m píxel) del 
plan PNOA que, además de las bandas RGB, tienen otra infrarroja, han sido utilizadas para realizar la clasificación de las formas fluviales mediante Support Vector Machine y posteriormente se han efectuado varios análisis de transición entre los años de estudio (2017-2020). El estudio se ha centrado en tres tramos de la rambla de Cervera (Castelló), curso efímero de gravas. Los resultados permiten caracterizar los cambios anuales en las formas indicadoras de actividad geomorfológica y el grado de recuperación morfo-sedimentaria en respuesta a la dinámica del flujo, respuestas condicionadas por la geometría del corredor fluvial y por impactos humanos. Se observó que en todos los tramos había zonas donde la superficie de cauce activo y la de gravas sin vegetación experimentaron cierta recuperación, debido a la propia actividad morfo-sedimentaria natural. A su vez, esa apertura de espacios favorece la colonización de vegetación pionera, que estabiliza las superficies del lecho más dinámicas.

En el segundo bloque, que analiza casos de estudio de cambios derivados de impactos, la propuesta de Prats-Puntí et al. (2021) estudia los efectos de las obras de ingeniería en el río Llobregat en la regresión de su delta, que ha retrocedido hasta 800 metros desde finales del siglo xix. En esta investigación se ha establecido un método para conocer el transporte sólido de fondo a partir de la información de la morfología del río en el pasado. El artículo compara los cálculos con mediciones del volumen de sedimento atrapado en la nueva desembocadura. Los encauzamientos realizados en los últimos setenta años en el curso bajo del Llobregat para el paso de infraestructuras han disminuido la disponibilidad de sedimento en los últimos $30 \mathrm{~km}$ del río, pero esta no es la causa principal de la reducción de la aportación de sedimento grosero hacia la costa. La construcción de numerosas presas en el curso medio durante el siglo xix y la reducción de la frecuencia de crecidas generadoras de las grandes aportaciones de sedimento serían las responsables del retroceso de la línea de la costa del delta desde finales del siglo xix y a lo largo del xx. La retención de sedimento en los embalses es menos relevante que la regulación que estos ejercen en el régimen de caudales. Consecuentemente, para proveer arena en las playas del delta del Llobregat sería más eficiente la retirada de presas y el ensanchamiento del cauce desmantelando encauzamientos, que traspasar sedimento desde los grandes embalses.

Ibisate et al. (2021) analizan la evolución y relación entre los usos del suelo, el cauce activo y las actuaciones realizadas en el último medio siglo en las cuencas de los ríos Oiartzun y Oria (Gipuzkoa) en los últimos sesenta años. Han constatado una importante reducción de la superficie del cauce activo, con estrechamiento e incremento de la vegetación, coincidiendo en el tiempo con un incremento de la superficie arbórea y arbustiva y suelos urbanizados en ambas cuencas, en detrimento de usos de praderas. Durante el mismo periodo se han visto ocupadas las escasas llanuras de inundación funcionales que restaban y se han acometido muchas obras de defensa y modificación de los cauces. Todo ello ha afectado a la superficie del cauce activo y es, probablemente, el factor más relevante de su ajuste. Se han podido identificar dos dinámicas diferentes: en áreas semiurbanas se ha producido un descenso de la actividad del cauce, menor número de barras, estrechamiento del cauce y mayor vegetación; y en áreas muy urbanizadas se ha constatado en general una disminución de la superficie del cauce activo, con un timing diferente según el periodo de urbanización. Así, a pesar de que existe un aumento de la superficie arbórea y arbustiva, simultáneo al retroceso de la superficie del cauce activo, las actuaciones directas en el cauce (modificación de trazados, construcción de defensas, ocupación de cauces, etc.) 
han repercutido en mayor medida a la disminución de la superficie fluvial. La reducción en el cauce activo ha conllevado su incisión, apreciable especialmente en las zonas con defensas. Pero la respuesta en el cauce no es sincrónica en todos los puntos de la cuenca, tal y como se aprecia en la respuesta de los distintos afluentes.

Marco et al. (2021) abordan el aprovechamiento tradicional de las aguas de avenida mediante presas de derivación y complejas redes de boqueras, que generó un milenario sistema de organización hidrogeomorfológica en las cuencas vertientes del sureste de la Península Ibérica. Tal fue su implantación e importancia territorial que, durante siglos, en barrancos y ramblas se desarrolló una dinámica ecoantrópica en la que las avenidas fluviales se convirtieron en un valioso recurso para la agricultura (sistema de riego de turbias), reduciendo su peligrosidad, pues se detraían importantes caudales desde las ramblas. El rápido abandono de estos sistemas de laminación durante el siglo xx, junto con la alteración de los cursos fluviales y los intensos cambios de usos del suelo, ha incrementado la peligrosidad de estas ramblas. El estudio se centra en el último tramo de la rambla de Abanilla-Benferri (Alacant), que constituye un ejemplo paradigmático de estos procesos territoriales. A partir de un detallado trabajo de búsqueda y reinterpretación de la cartografía y documentación histórica, se aporta una primera aproximación histórico-geomorfológica sobre su funcionamiento en el pasado y las consecuencias del abandono de este sistema de riego tradicional.

En el tercer bloque de trabajos, que pone el acento en las implicaciones para la gestión, el artículo de Batalla (2021) reflexiona sobre la importancia del análisis y el conocimiento de la dinámica morfosedimentaria para la conservación de la estructura y el funcionamiento de los cauces fluviales y, en su caso, para su rehabilitación. Por ejemplo, pueden definir umbrales de cambio irreversibles en medios fluviales poco o no alterados y programas de reconexión lateral y longitudinal en medios muy alterados. El autor señala que la investigación fluvial deberá avanzar principalmente en tres frentes. El primero debe aglutinar el conocimiento de ciencias complementarias o auxiliares con el fin de profundizar en el análisis del transporte de sedimento y procesos fluviales asociados (morfodinámica, hidráulica de los flujos, estructura del cauce) en todo tipo de cursos a través de técnicas avanzadas de medición y modelización. El segundo frente es el estudio mediante trabajo de campo, análisis y modelización de procesos hidrosedimentarios en ríos regulados, especialmente de grandes cuencas, con objetivos de gestión ambiental, como por ejemplo para el diseño de crecidas de mantenimiento. En tercer término, hay que trabajar en el conocimiento de las interacciones entre la hidráulica del flujo, el transporte de sedimento y la estructura del cauce (balance de masas y hábitat físico) con las comunidades bentónicas de macroinvertebrados y peces (estado ecológico), lo que permitiría entender mejor las relaciones biofísicas o ecogeomorfológicas de los sistemas fluviales.

Benito et al. (2021) abordan el problema de la incertidumbre sobre los efectos del cambio climático en la peligrosidad de las inundaciones. El caso de estudio es la rambla de la Viuda (Castelló), donde se ha aplicado un análisis de caudales máximos (recurrencia de cien años) desde proyecciones de precipitación extrema (escenario RCP 8.5) con modelos de clima regionales (Eurocordex), convertidos en caudal instantáneo mediante el modelo distribuido TETIS. Ante la elevada variabilidad de los resultados, se han analizado series largas de inundaciones obtenidas a partir de registros históricos y 
sedimentarios (paleoinundaciones) en momentos de calentamiento relativo, que han permitido obtener los caudales máximos registrados secularmente y se han analizado conjuntamente con registros instrumentales. Los datos de paleocrecidas han permitido validar los datos de los modelos de clima e hidrológicos para los cuantiles altos. La metodología aplicada -esta aproximación local basada en el conocimiento de los extremos combinando modelos y datos pasados- genera conocimiento y series de datos que permiten identificar incertidumbres y certezas, por lo que permite avanzar en la adaptación al cambio climático y ofrece también una evidencia clara de sucesos de inundación que posibilitan la recuperación de la cultura del riesgo.

Vidal-Abarca et al. (2021) exploran desde una perspectiva ecosocial los beneficios de los ríos secos o cursos efímeros para el bienestar humano. La ausencia permanente de agua en estos cauces constituye la principal razón por la que gestores y sociedad civil, en general, los consideran inútiles e improductivos, con lo que son ecosistemas enormemente impactados. Los autores revisan la diversidad de servicios ecosistémicos que estos sistemas fluviales proporcionan al bienestar humano y detectan los impulsores directos e indirectos de cambio, claves que afectan a la capacidad de estos ríos para generar un flujo sostenible de servicios. El trabajo incluye un estudio de la percepción social que la ciudadanía de la región de Murcia tiene sobre los ríos secos aplicando la técnica de libre listado o freelisting. Los resultados muestran que estos ecosistemas contribuyen al bienestar humano, no tanto por su alta capacidad de proveer servicios ecosistémicos, sino sobre todo por la fuerte relación y colaboración que a lo largo de la historia ha existido entre las comunidades que viven en torno a estos ecosistemas y los recursos que proveen. Sin embargo, esta coproducción de beneficios para el bienestar humano que se establece entre ambos sistemas es especialmente sensible a cambios políticos, económicos, sociales y ambientales como el cambio climático. De hecho, los cambios socioeconómicos están alterando los modelos de sostenibilidad que mantenían un alto grado de resiliencia de estos socioecosistemas. Pero la consulta a la ciudadanía reveló paradójicamente que, aunque el conocimiento actual sobre los ríos secos no es completo y su utilidad está infravalorada, existe un valor sociocultural fuertemente arraigado.

\section{Agradecimientos}

Este trabajo ha sido financiado por los proyectos del Ministerio de Economía y Competitividad CGL2017-86839-C3-1-R y CGL2017-84625-C2-1-R, y por el proyecto del Ministerio de Ciencia e Innovación PID2020-116537RB-I00.

\section{REFERENCIAS}

Batalla, R. J. (2003). Sediment deficit in rivers caused by dams and instream gravel mining: A review with examples from NE Spain. Cuaternario y Geomorfología, 17(3), 79-91.

Batalla, R. J. (2021). Reflexión sobre dinámica morfosedimentaria. Implicaciones para la gestión fluvial en un contexto de cambio global. Cuadernos de Geografía de la Universitat de València, 107. https://doi.org/10.7203/CGUV.107.213372 
Benito, G., Thorndycraft, V. R., Rico, M., Sánchez-Moya, Y., \& Sopeña, A. (2008). Palaeoflood and floodplain records from Spain: Evidence for long-term climate variability and environmental changes. Geomorphology, 101, 68-77. https://doi.org/10.1016/j. geomorph.2008.05.020

Benito, G., Beneyto, C., Aranda, J. A., Machado, M. J., Francés, F., \& Sánchez-Moya, Y. (2021). Inundaciones y Cambio Climático: certezas e incertidumbres en el camino a la adaptación. Cuadernos de Geografía de la Universitat de València, 107. https:/doi. org/10.7203/CGUV.107.21424

Calle, M., Alho, P., \& Benito, G. (2017). Channel dynamics and geomorphic resilience in an ephemeral Mediterranean river affected by gravel mining. Geomorphology, 285, 333 346. https://doi.org/10.1016/j.geomorph.2017.02.026

Clarke, S. J., Bruce-Burgess, L., \& Wharton, G. (2003). Linking form and function: towards an eco-hydromorphic approach to sustainable river restoration. Aquatic Conservation. Marine and Freshwater Ecosystems, 13, 439-450.

Comisión Europea (2000). Directive 2000/60 EC of the European Parliament and of the Council of 23 October 2000 establishing a framework for Community action in the field of water policy. Official Journal L 327, 22/12/2000, 73 pp.

Díaz Alba, D., Martínez Fernández, V., \& García de Jalón, D. (2021). Seguimiento ambiental en riberas mediante tecnología LiDAR. Cuadernos de Geografía de la Universitat de València, 107. https://doi.org/10.7203/CGUV.107.21240

Díez Herrero, A., Ballesteros, J. A., Génova, M. M., Pérez López, R., \& Garrote, J. (2021). Aportaciones de la dendrogeomorfología y la liquenometría al análisis de avenidas torrenciales pretéritas en sistemas fluviales. Cuadernos de Geografía de la Universitat de València, 107. https://doi.org/10.7203/CGUV.107.21259

Dufour, S. \& Piégay, H. (2009). From the myth of a lost paradise to targeted river restoration: forget natural references and focus on human benefits. River research and applications, 25(5), 568-581. https://doi.org/10.1002/rra.1239

García, J. H., Ollero, A., Ibisate, A., Fuller, I. C., Death, R. G., \& Piégay, H. (2021). Promoting fluvial geomorphology to "live with rivers" in the Anthropocene Era. Geomorphology, 380, https://doi.org/10.1016/j.geomorph.2021.107649

García, J. H. \& Pérez Alberti, A. (2021). Aproximación a la identificación y caracterización de ríos en roca a escala regional mediante variables topo-geomorfológicas (Galicia, Noroeste de la Península Ibérica). Cuadernos de Geografía de la Universitat de València, 107. https://doi.org/10.7203/CGUV.107.21426

García-Ruiz, J. M. \& Lana-Renault, N. (2011). Hydrological and erosive consequences of farmland abandonment in Europe, with special reference to the Mediterranean region: A review. Agriculture, Ecosystems and Environment, 140, 317-338. https://doi.org/10.1016/j. agee.2011.01.003 
García Ruiz, J. M., López Moreno, J. I., Lasanta, T., Vicente, S. M., González Sampériz, P., Valero, B. L., Sanjuán, Y., Beguería, S., Nadal, E., Lana-Renault, N., \& Gómez Villar, A. (2015). Los efectos geoecológicos del cambio global en el Pirineo central español: una revisión a distintas escalas espaciales y temporales. Pirineos. Revista de Ecología de Montaña, 170, http://dx.doi.org/10.3989/Pirineos.2015.170005.

García de Jalón, D., Martínez-Fernández, V., Fazelpoor, K., \& González, M. (2020). Vegetation encroachment ratios in regulated and non-regulated Mediterranean rivers (Spain): An exploratory overview. Journal of Hydro-environment Research, 30, 35-44. https://doi.org/10.1016/j.jher.2019.11.006

Garófano-Gómez, V., Martínez-Capel, F., Bertoldi, W., Gurnell, A., Estornell, J., \& SeguraBeltrán, F. (2013). Six decades of changes in the riparian corridor of a Mediterranean river: a synthetic analysis based on historical data sources. Ecohydrology, 6(4), 536-553. https://doi.org/10.1002/eco.1330

Gómez Gutiérrez, A., Conesa-García, C., Ibisate, A., Pérez-Cutillas, P., Portillo-Grau, D., \& Segura-Méndez, F. J. (2021). Potencial de las imágenes aéreas históricas y la fotogrametría automatizada para elaborar modelos 3D de cauces efímeros mediterráneos y cuantificar cambios morfológicos. Cuadernos de Geografía de la Universitat de València, 107. https://doi.org/10.7203/CGUV.107.21119

Gurnell, A. M., Surian, N., \& Zanoni, L. (2009). Multi-thread river channels: a perspective on changing European alpine river systems. Aquatic Sciences. Research Across Boundaries, 71(3), 253-265.

Gurnell, A. M., Bertoldi, W., \& Corenblit, D. (2012). Changing river channels: the roles of hydrological processes, plants and pioneer fluvial landforms in humid temperate, mixed load, gravel bed rivers. Earth-Science Reviews, 111(1-2), 129. https://doi.org/10.1016/j. earscirev.2011.11.005

Gurnell, A. M., Corenblit, D., García de Jalón, D., González del Tánago, M., Grabowski, R. C., O'Hare, M. T., \& Szewczyk, M. (2015). A conceptual model of vegetation-hydrogeomorphology interactions within river corridors. River Research and Applications, 32, 142-163. https://doi.org/10.1002/rra.2928

Ibisate, A., Acín, V., Granado, D., \& Sánez de Olazagoitia, A. (2021). Cambios de usos del suelo y relación con cambios en cauces cantábricos (Oiartzun y Oria). Cuadernos de Geografía de la Universitat de València, 107. https://doi.org/10.7203/CGUV.107.21625

Hooke, J. M. \& Mant, J. (2002). Morpho-dynamics of ephemeral streams. En Bull, L. \& Kirkby, M. (eds.). Dryland rivers: hydrology and geomorphology of semi-arid channels (pp. 173-204). Chichester: John Wiley \& Sons.

Liébault, F. \& Piégay, H. (2001). Assessment of channel changes due to long-term bedload supply decrease, Roubion River, France. Geomorphology, 36(3), 167-186. https://doi. org/10.1016/S0169-555X(00)00044-1 
Liébault, F. \& Piégay, H. (2002). Causes of 20th century channel narrowing in mountain and piedmont rivers of Southeastern France. Earth Surface and Landforms, 27, 425-444. https://doi.org/10.1002/esp.328

Llasat, M. C. (2021). Floods evolution in the Mediterranean region in a context of climate and environmental change. Cuadernos de Investigación Geográfica, 47(1), 13-32 https:// doi.org/10.18172/cig.4897

Macklin, M. G. \& Woodward, J. C. (2009). River system and environmental change. En Woodward, J. C. (ed.). The Physical Geography of the Mediterranean (pp. 319-352). Oxford University Press.

Martín-Vide, J. P., Ferrer-Boix, C., \& Ollero, A. (2010). Incision due to gravel mining: modeling a case study from the Gállego River, Spain. Geomorphology, 117(3-4), 261-271. https://doi.org/10.1016/j.geomorph.2009.01.019

Martínez-Fernández, V., González del Tánago, M., Maroto, J., \& García de Jalón, D. (2016). Fluvial corridor changes over time in regulated and non-regulated rivers (Upper Esla River, NW Spain). River Research and Applications, 33(2), 214-223. https://doi. org/10.1002/rra.3032

Marco, J. A., Giménez, P., \& Prieto, A. (2021). Aprovechamiento tradicional de las aguas de avenida y transformaciones de los sistemas fluviales del sureste de la Península Ibérica: la Rambla de Abanilla-Benferri. Cuadernos de Geografía de la Universitat de València, 107. https://doi.org/10.7203/CGUV.107.21333

Ollero, A. (2011). Restauración fluvial: principios, dificultades y propuestas. La perspectiva del CIREF. En I Congreso Ibérico de Restauración Fluvial (pp. 36-45). León: MARM, CHD y CIREF.

Ollero, A. (2015). Guía metodológica sobre buenas prácticas en restauración fluvial (manual para gestores). Zaragoza: Contrato de río del Matarraña, ECODES.

Ollero, A. (2017). Hidrogeomorfología y geodiversidad: el patrimonio fluvial. Zaragoza: Centro de Documentación del Agua y del Medio Ambiente.

Ollero, A. (2020). Sección de desagüe, alteración de la geomorfología en cauces aluviales y restauración fluvial. RestauraRios, 1, https://doi.org/10.51443/Restaurarios.2020.01

Ollero, A., Ibisate, A., Granado, D., \& Real de Asua, R. (2015). Channel responses to global change and local impacts: perspectives and tools for floodplain management (Ebro River and tributaries, NE Spain). En Hudson, P. F. \& Middelkoop, H. (eds.). Geomorphic approaches to integrated floodplain management of lowland fluvial systems in North America and Europe (pp. 27-52). Nueva York: Springer.

Ollero, A., García, J. H., Ibisate, A., Ballarín, D., Sáenz de Olazagoitia, A., \& Sánchez Fabre, M. (2020). La Geografía Física en la caracterización, evaluación y restauración hidromorfológica fluvial. En España, puente entre continentes (pp. 167-178). Estambul: Aportación Española al 34 Congreso de la Unión Geográfica Internacional. 
Ollero, A., Conesa-García, C., \& Vidal-Abarca, M. R. (coords.) (2021). Buenas prácticas en gestión y restauración de cursos efímeros mediterráneos: resiliencia y adaptación al cambio climático. Murcia: Universidad de Murcia. https://doi.org/10.6018/editum.2900

Prats-Puntí, A., Martín Vide, J. P., \& Ferrer-Boix, C. (2021). Regressió del delta del Llobregat. Efecte de les obres d'enginyeria al riu d'ençà del segle XIX. Cuadernos de Geografía de la Universitat de València, 107. https://doi.org/10.7203/CGUV.107.21307

Rabanaque, M. P., Martínez Fernández, V., \& Benito, G. (2021). Caracterización diacrónica de unidades morfosedimentarias en cauces efímeros mediante análisis automatizado de ortofotografías y técnicas de machine learning. Cuadernos de Geografía de la Universitat de València, 107. https://doi.org/10.7203/CGUV.107.21218

Rhoads, B. L. (2020). River dynamics: geomorphology to support management. Cambridge University Press.

Sanchis-Ibor, C., Segura-Beltrán, F., \& Almonacid-Caballer, J. (2017). Channel forms recovery in an ephemeral river after gravel mining (Palancia River, Eastern Spain). Catena, 158, 357-370. https://doi.org/10.1016/j.catena.2017.07.012

Sanchis-Ibor, C., Segura-Beltrán, F., \& Navarro-Gómez, A. (2019). Channel forms and vegetation adjustment to damming in a Mediterranean gravel-bed river (Serpis River, Spain). River Research and Applications, 35(1), 37-47. https://doi.org/10.1002/rra.3381

Sanchis-Ibor, C. \& Segura-Beltran, F. (2020). Procesos de colonización vegetal en ramblas: crecimiento y destrucción de parches y alineaciones. En Farinós, J., Escribano, J., Peñarrubia, M. P., Serrano, J., \& Asins, S. (eds.). Desafíos y oportunidades de un mundo en transición: una interpretación desde la Geografía (pp. 161-171). Universitat de València.

Sandercock, P. J., Hooke, J. M., \& Mant, J. M. (2007). Vegetation in dryland river channels and its interaction with fluvial processes. Progress in Physical Geography, 31, 107-129. https://doi.org/10.1177/0309133307076106

Sear, D. A. \& Newson, M. D. (2003). Environmental change in river channels: a neglected element. Towards geomorphological typologies, standards and monitoring. The Science of the Total Environment, 310, 17-23. https://doi.org/10.1016/S0048-9697(02)00619-8

Scorpio, V. \& Piégay, H. (2021). Is afforestation a driver of change in italian rivers within the Anthropocene era? Catena, 198, https://doi.org/10.1016/j.catena.2020.105031

Segura-Beltran, F. (2014). Sobre la restauració fluvial i la complexitat dels rius efímers: algunes consideracions crítiques. Cuadernos de Geografía de la Universitat de València, 95-96, $101-147$.

Segura-Beltran, F. \& Sanchis-Ibor, C. (2013). Assessment of channel changes in a Mediterranean ephemeral stream since the early twentieth century. The Rambla de Cervera, eastern Spain. Geomorphology, 201, 199-214. https://doi.org/10.1016/j. geomorph.2013.06.021 
Segura-Beltran, F. \& Sanchis-Ibor, C. (2018). Estrechamiento de cauces y cambio de patrón en cursos fluviales mediterráneos como consecuencia del cambio global en las últimas décadas: indicadores geomorfológicos. En García, C., Gómez-Pujol, L., Morán-Tejeda, E., \& Batalla, R. J. (eds.). Geomorfología del Antropoceno. Efectos del Cambio Global sobre los procesos geomorfológicos (pp. 395-398). Palma: Universitat de les Illes Balears, Sociedad Española de Geomorfología.

Segura-Beltran, F. \& Sanchis-Ibor, C. (2020). La incisión como efecto de los cambios ambientales en ríos efímeros. En Farinós, J., Escribano, J., Peñarrubia, M. P., Serrano, J., \& Asins, S. (eds.). Desafíos y oportunidades de un mundo en transición: una interpretación desde la Geografía (pp. 145-160). Universitat de València.

Segura-Beltran, F., Sanchis-Ibor, C., \& Ollero, A. (2021). Restauración hidrogeomorfológica en ramblas y ríos semipermanentes. En Thomsen, A., Farinós, J. \& Perero, E. (coords.). Soluciones ante los riesgos climáticos en ríos y costas (pp. 207-215). Fundación CONAMA. http://www.conama.org/conama/download/files/conama2020//STs\%202020/30_final.pdf

Surian, N. \& Rinaldi, M. (2003). Morphological response to river engineering and management in alluvial channels in Italy. Geomorphology, 50, 307-326. https://doi.org/10.1016/ S0169-555X(02)00219-2

Surian, N., Ziliani, L., Comiti, F., Lenzi, M. A., \& Mao, L. (2009). Channel adjustments and alteration of sediment fluxes in gravel-bed rivers of north-eastern Italy: potentials and limitations for channel recovery. River Research and Applications, 25(5), 551-567. https:// doi.org/10.1002/rra.1231

Vidal-Abarca, M. R., Nicolás, N., \& Suárez, M. L. (2021). Explorando los beneficios de los ríos secos para el bienestar humano: una perspectiva social. Cuadernos de Geografía de la Universitat de València, 107. https://doi.org/10.7203/CGUV.107.20783

Williamson, C. E., Dodds, W., Kratz, T. K., \& Palmer, M. A. (2008). Lakes and streams as sentinels of environmental change in terrestrial and atmospheric processes. Frontiers in Ecology and the Environment, 6(5): 247-254. https://doi.org/10.1890/070140

Wyżga, B., Zawiejska, J., Radecki-Pawlik, A., \& Hajdukiewicz, H. (2012). Environmental change, hydromorphological reference conditions and the restoration of Polish Carpathian rivers. Earth Surface Processes and Landforms, 37, 1213-1226. https://doi. org/10.1002/esp.3273 
